\title{
Angular analysis of bottom-flavored hadron production in semileptonic decays of polarized top quarks
}

\author{
Bernd A. Kniehlø* \\ II. Institut für Theoretische Physik, Universität Hamburg, \\ Luruper Chaussee 149, 22761 Hamburg, Germany \\ S. Mohammad Moosavi Nejad $\odot^{\dagger}$ \\ Faculty of Physics, Yazd University, P.O. Box 89195-741 Yazd, Iran
}

(Received 10 December 2020; accepted 13 January 2021; published 22 February 2021)

\begin{abstract}
We study the inclusive production of bottom-flavored hadrons from semileptonic decays of polarized top quarks at next-to-leading order in QCD using fragmentation functions recently determined from a global fit to $e^{+} e^{-}$data. We provide the relevant differential decay widths at parton level in analytic form. These results fill an important gap in the theoretical interpretation of recent measurements of the top-quark polarization and the $t \bar{t}$ spin correlations using dilepton final states in proton-proton collisions at the CERN Large Hadron Collider. As an illustration, we study the distributions in the scaled bottom-hadron energy of the polarized-top-quark decay widths for different $W$-boson helicities.
\end{abstract}

DOI: $10.1103 /$ PhysRevD.103.034015

\section{INTRODUCTION}

The top quark $t$ of the standard model (SM) is the heaviest known elementary particle. Due to its high mass, it plays a crucial role in testing the electroweak symmetry breaking mechanism and in searching for new physics beyond the SM. The precise determination of its properties, including its mass $m_{t}$ and total decay width $\Gamma_{t}$, and its process-dependent features, like its polarization or the correlation of its spin with that of a co-produced antitop quark, is of prime importance. The latter quantities are particularly sensitive probes of deviations from the SM and allow us to constrain, e.g., the anomalous chromoelectric and chromomagnetic dipole moments of the top quark. The top-quark lifetime $\tau_{t}=\hbar / \Gamma_{t} \approx 5 \times 10^{-25} \mathrm{~s}$ [1] is shorter than the typical time scale of quantum chromodynamics (QCD) $\hbar / \Lambda_{\mathrm{QCD}} \approx 10^{-24} \mathrm{~s}$ and much shorter than the spin correlation time scale $\hbar m_{t} / \Lambda_{\mathrm{QCD}}^{2} \approx 10^{-21} \mathrm{~s}$, where $\Lambda_{\mathrm{QCD}}$ is the asymptotic scale parameter of QCD. Therefore, the top quark decays before it can hadronize, and its full spin information is preserved during its decay process and fully encoded in the angular distribution of its decay products.

\footnotetext{
*kniehl@desy.de

†mmoosavi@yazd.ac.ir
}

Published by the American Physical Society under the terms of the Creative Commons Attribution 4.0 International license. Further distribution of this work must maintain attribution to the author(s) and the published article's title, journal citation, and DOI. Funded by SCOAP.
The top-quark polarization and the $t \bar{t}$ spin correlations have recently been measured using dilepton final states in Run 2 at the CERN Large Hadron Collider (LHC) with center-of-mass energy $\sqrt{s}=13 \mathrm{TeV}$, by the ATLAS [2] and CMS [3] Collaborations. Intriguingly, ATLAS found a deviation of 3.8 standard deviations $(\sigma)$ from the SM prediction of the asymmetry $A_{\left|\Delta \phi_{\ell \ell}\right|}$ of the distribution in the azimuthal angular difference $\Delta \phi_{\ell \ell}$ of the decay leptons. This deviation was confirmed by CMS, albeit with a smaller significance of about $2 \sigma$. These analyses are relying on the factorization of the squared matrix element of the full process, $\left|\mathcal{M}\left(q \bar{q} / g g \rightarrow t \bar{t} \rightarrow b \ell^{+} \nu \bar{b} \ell^{-} \bar{\nu}\right)\right|^{2} \propto \rho \times R \times \bar{\rho}$, into the spin density matrices $R, \rho$, and $\bar{\rho}$ for on-shell $t \bar{t}$ hadroproduction and semileptonic $t$ and $\bar{t}$ decays, respectively, via the narrow-width approximation. While $R$ is treated in Refs. [2,3] at next-to-leading order (NLO) in QCD [4,5], $\rho$ and $\bar{\rho}$ are only modeled at leading order (LO) using the program package MADSPIN [6]. Moreover, the formation of bottom-flavored hadrons is not taken into account within the rigorous framework of the QCD parton model with fragmentation functions (FFs) whose universality is guaranteed by the factorization theorem [7]. It is an urgent matter to clarify in how far the observed deviations may be related to a lack of precision in the theoretical treatment of the semileptonic $t$ and $\bar{t}$ decays.

It is the purpose of the present paper to provide theoretical input needed to fill this gap. Specifically, we calculate the partial width of the inclusive decay $t(\uparrow) \rightarrow$ $b W^{+}(\uparrow) \rightarrow B l^{+} \nu_{l}+X$, where $B$ generically denotes a bottom-flavored hadron, at NLO in QCD allowing for top-quark polarization and definite $W$-boson helicity and 
properly accounting for parton-to-hadron fragmentation and finite-hadron-mass effects. By doing so, we generalize our previous work [8], where the top-quark spin was averaged over.

The general-mass variable-flavor-number scheme (GM-VFNS), which has been elaborated for inclusive heavy-flavored-hadron production in $e^{+} e^{-}$annihilation [9], two-photon collisions [10], photoproduction [11], and hadroproduction [12-15], provides an ideal theoretical framework also here. However, owing to the large mass hierarchy $m_{b} \ll m_{t}$, finite- $m_{b}$ corrections are expected to be negligible in the case at hand. This expectation was actually confirmed in Ref. [8], by a comparative analysis of the partial width of the decay $t \rightarrow B+W^{+}$in the GM-VFNS and the zero-mass variable-flavor-number scheme (ZMVFNS), where bottom is included among the massless quark flavors. In fact, the finite- $m_{b}$ corrections were found to be much smaller than the contribution from gluon fragmentation. Therefore, we will adopt the ZM-VFNS in the following. However, we will include finite- $m_{B}$ effects, which modify the relations between partonic and hadronic variables and reduce the available phase space, as explained in Sec. 2 of Ref. [8].

In Ref. [8], we adopted the $B$ FFs from Ref. [16], which were determined at NLO in the ZM-VFNS through a joint fit to $e^{+} e^{-}$annihilation data taken by ALEPH [17] and OPAL [18] at CERN LEP1 and by SLD [19] at SLAC SLC. Specifically, the power ansatz $D_{b}\left(z, \mu_{F}^{\text {ini }}\right)=N z^{\alpha}(1-z)^{\beta}$ was used as the initial condition for the $b \rightarrow B$ FF at factorization scale $\mu_{F}^{\text {ini }}=m_{b}=4.5 \mathrm{GeV}$, while the gluon and light-quark FFs were generated via the DokshitzerGribov-Lipatov-Altatelli-Parisi (DGLAP) [20-22] evolution. In Ref. [23], the analysis of Ref. [16] was updated by including the data taken by DELPHI [24] at CERN LEP1, which were published after Ref. [16], working both at NLO and next-to-next-to-leading order (NNLO) with the same theoretical assumptions. In our numerical analysis, we employ the new $B$ FFs from Ref. [23].

A similar analysis, albeit without fragmentation and finite- $m_{B}$ effects, was reported in Refs. [25,26]. Our analysis provides an independent check of analytic results presented therein. A related NLO analysis with a different treatment of the final state, for bottom jets instead of bottom hadrons, was performed in Ref. [27], leading to results that, unfortunately, cannot be compared with ours in any straightforward way. Recently, the analysis of Refs. [25,26] has been extended to NNLO in QCD using the optical theorem [28]. Due to the totally inclusive treatment of the hadronic part of the final state, this result does not allow for the implementation of FFs.

This paper is organized as follows. In Sec. II, we list our parton-level results in analytic form, relegating lengthy formulas to the Appendix. In Sec. III, we present our numerical analysis. In Sec. IV, we summarize our conclusions.

\section{ANALYTIC RESULTS}

We work at NLO in the ZM-VFNS, implemented in the modified minimal-subtraction ( $\overline{\mathrm{MS}}$ ) scheme, and consider the decay process

$$
\begin{aligned}
t\left(p_{t}, s\right) & \rightarrow b\left(p_{b}\right)+W^{+}\left(p_{W}, \lambda\right)\left(+g\left(p_{g}\right)\right) \\
& \rightarrow B\left(p_{B}\right)+\ell^{+}\left(p_{\ell}\right)+\nu_{\ell}\left(p_{\nu}\right)+X,
\end{aligned}
$$

where $X$ collectively denotes the unobserved final-state hadrons and the four-momentum, spin, and helicity assignments are indicated in parentheses. We have $s= \pm 1 / 2$ and $\lambda=0, \pm 1$. The gluon in Eq. (1) contributes to the real radiation at NLO. Both the $b$ quark and the gluon may hadronize into the $B$ hadron. For simplicity, we employ the narrow-width approximation, where $p_{W}^{2}=m_{W}^{2}$ and small terms of order $\mathcal{O}\left(\Gamma_{W}^{2} / m_{W}^{2}\right)$ are neglected. As mentioned in Sec. I, we put $m_{b}=0$, but keep $m_{B}$ finite. In the top-quark rest frame, the $b$ quark, gluon, and $B$ hadron have energies $E_{i}=p_{t} \cdot p_{i} / m_{t}(i=b, g, B)$, which nominally range from $E_{b}^{\min }=E_{g}^{\min }=0$ and $E_{B}^{\min }=m_{B}$ to $E_{b}^{\max }=E_{g}^{\max }=$ $\left(m_{t}^{2}-m_{W}^{2}\right) /\left(2 m_{t}\right)$ and $E_{B}^{\max }=\left(m_{t}^{2}+m_{B}^{2}-m_{W}^{2}\right) /\left(2 m_{t}\right)$, respectively. As in Ref. [8], we choose the scaling variable $z$ by setting $E_{B}=z E_{a}$, with $a=b, g$, in the range $0 \leq z \leq 1$. Introducing the scaled energies $x_{i}=E_{i} / E_{b}^{\max }$ $(i=b, g, B)$, we then have $m_{B} / E_{b}^{\max } \leq x_{B} \leq x_{a} \leq 1$. An alternative definition of the scaling variable, in terms of light-cone variables, is discussed in Sec. 3 of Ref. [8], to where we refer the interested reader.

We implement the polarization of the top quark by writing its (average) spin four-vector in its rest frame as $s_{t}^{\mu}=P\left(0, \sin \theta_{P} \cos \phi_{P}, \sin \theta_{P} \sin \phi_{P}, \cos \theta_{P}\right)$, where $P$ is the magnitude of the top-quark polarization, taking values in the range $0 \leq P \leq 1$. Here, it is understood that the $z$ axis is chosen to point along the $W$-boson flight direction, so that $s_{t} \cdot p_{W}=-P\left|\vec{p}_{W}\right| \cos \theta_{P}$. To describe the leptonic decay of the $W$ boson, we boost into the rest frame of the latter, which leaves the $z$ axis invariant, and define the charged-lepton four-momentum to be $p_{\ell}^{\mu}=E_{\ell}(1, \sin \theta \cos \phi, \sin \theta \sin \phi, \cos \theta)$. The polar angles $\theta_{P}$ and $\theta$, which appear in our final results, are also illustrated in Fig. 1.

We wish to calculate the triply differential partial decay width $d^{3} \Gamma /\left(d x_{B} d \cos \theta d \cos \theta_{P}\right)$ of process (1). Analogously to Eq. (3) in Ref. [8], we have

$$
\begin{aligned}
& \frac{d^{3} \Gamma}{d x_{B} d \cos \theta d \cos \theta_{P}} \\
& =\sum_{a=b, g} \int_{x_{B}}^{1} \frac{d x_{a}}{x_{a}} \frac{d^{3} \hat{\Gamma}_{a}}{d x_{a} d \cos \theta d \cos \theta_{P}}\left(\mu_{R}, \mu_{F}\right) D_{a}\left(\frac{x_{B}}{x_{a}}, \mu_{F}\right),
\end{aligned}
$$

where $\mu_{R}$ is the renormalization scale, $\mu_{R}$ is the factorization scale, $D_{a}\left(z, \mu_{F}\right)$ is the $a \rightarrow B \mathrm{FF}$, and 


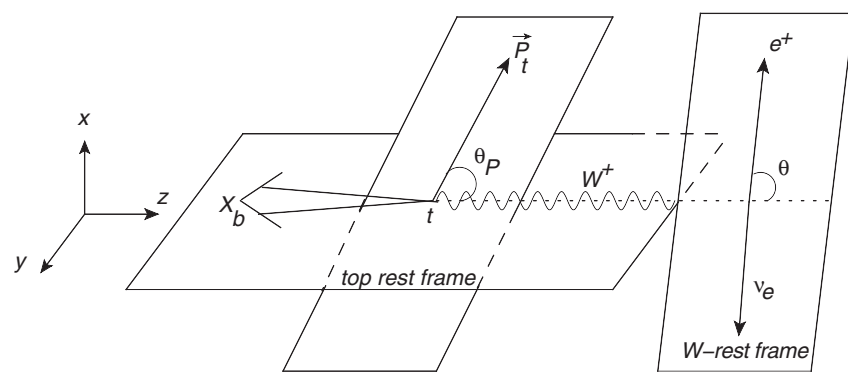

FIG. 1. Definitions of the polar angle $\theta_{P}$ of the top-quark polarization three-vector in the top-quark rest frame and of the polar angle $\theta$ of the charged-lepton three-momentum in the $W$ boson rest frame. In both cases, the $z$ axis is chosen to point along the $W$-boson three-momentum in the top-quark rest frame.

$\frac{d^{3} \hat{\Gamma}_{a}}{d x_{a} d \cos \theta d \cos \theta_{P}}=\frac{1}{2}\left(\frac{d^{2} \hat{\Gamma}_{a}^{\mathrm{unpol}}}{d x_{a} d \cos \theta}+P \frac{d^{2} \hat{\Gamma}_{a}^{\mathrm{pol}}}{d x_{a} d \cos \theta} \cos \theta_{P}\right)$.

The factor $1 / 2$ on the right-hand side of Eq. (3) ensures that

$$
\int_{-1}^{1} d \cos \theta_{P} \frac{d^{3} \hat{\Gamma}}{d x_{a} d \cos \theta d \cos \theta_{P}}=\frac{d^{2} \hat{\Gamma}_{a}^{\text {unpol }}}{d x_{a} d \cos \theta} .
$$

Each $W$-boson helicity is featured by a characteristic $\theta$ dependence, which is encoded in the structure

$$
\begin{aligned}
\frac{d^{2} \hat{\Gamma}_{a}}{d x_{a} d \cos \theta}= & \frac{3}{8}(1+\cos \theta)^{2} \frac{d \hat{\Gamma}_{a}^{+}}{d x_{a}}+\frac{3}{8}(1-\cos \theta)^{2} \frac{d \hat{\Gamma}_{a}^{-}}{d x_{a}} \\
& +\frac{3}{4} \sin ^{2} \theta \frac{d \hat{\Gamma}_{a}^{0}}{d x_{a}} .
\end{aligned}
$$

This holds for both the unpolarized and polarized terms in Eq. (3). Notice that the $\theta$-dependent coefficients on the right-hand side of Eq. (5) are normalized so that, upon integration over $\cos \theta$, we have

$$
\frac{d \hat{\Gamma}_{a}}{d x_{a}}=\sum_{\lambda=-1}^{1} \frac{d \hat{\Gamma}_{a}^{\lambda}}{d x_{a}}
$$

At LO, we only have $a=b$, and $x_{b}=1$ is fixed, i.e., the $x_{b}$ dependence comes as a delta-function peak. Specifically, we have

$$
\begin{aligned}
& \hat{\Gamma}_{b, \mathrm{LO}}^{0, \text { unpol }}=\hat{\Gamma}_{b, \mathrm{LO}}^{0, \mathrm{pol}}=F(1-\omega)^{2}, \\
& \hat{\Gamma}_{b, \mathrm{LO}}^{-, \text {unpol }}=-\hat{\Gamma}_{b, \mathrm{LO}}^{-, \mathrm{pol}}=F(2 \omega)(1-\omega)^{2}, \\
& \hat{\Gamma}_{b, \mathrm{LO}}^{+, \text {unpol }}=\hat{\Gamma}_{b, \mathrm{LO}}^{+, \mathrm{pol}}=0,
\end{aligned}
$$

where $\omega=m_{W}^{2} / m_{t}^{2}$ and $F=G_{F} m_{t}^{3}\left|V_{t b}\right|^{2} B\left(W^{+} \rightarrow \ell^{+} \nu_{\ell}\right) /$ $(8 \pi \sqrt{2})$. Here, $G_{F}$ is Fermi's constant, $V_{i j}$ is the $i j$ element of the Cabibbo-Kobayashi-Matrix quark mixing (a)

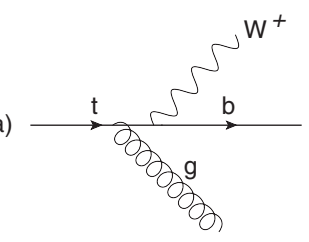

(c)

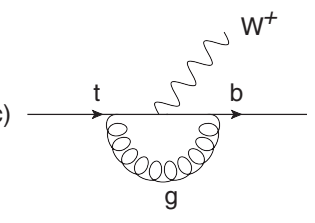

(b)

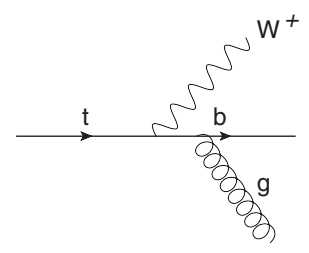

(d)

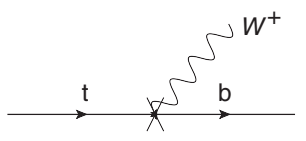

FIG. 2. Feynman diagrams contributing to the partial decay width of the process in Eq. (1) at NLO: (a) initial-state radiation; (b) final-state radiation; (c) vertex correction; and (d) combination of wave function renormalizations and vertex counterterm. The leptonic $W$-boson decay is not shown.

matrix [29,30], and $B\left(W^{+} \rightarrow \ell^{+} \nu_{\ell}\right)$ is the branching ratio of the leptonic $W$-boson decay mode considered. Neglecting the masses of the charged leptons and the first five quark flavors, we have

$$
\begin{aligned}
B\left(W^{+} \rightarrow \ell^{+} \nu_{\ell}\right) & =\frac{1}{3+N_{c} \sum_{\substack{i=u, c \\
j=d, s, b}}\left|V_{i j}\right|^{2}\left[1+3 C_{F} \alpha_{s}\left(\mu_{R}\right) /(4 \pi)\right]} \\
& \approx \frac{1}{9}\left[1-\frac{2 \alpha_{s}\left(\mu_{R}\right)}{3}\right]
\end{aligned}
$$

where we have included the NLO QCD correction, with color factors $N_{c}=3$ and $C_{F}=\left(N_{c}^{2}-1\right) /\left(2 N_{c}\right)=4 / 3$. In the last equality in Eq. (8), we have approximated $V_{i j} \approx \delta_{i j}$. At LO, the top-quark spin is passed on to the bottom quark for $\lambda=0$, while it is flipped for $\lambda=-1$; the case $\lambda=+1$ is forbidden by angular-momentum conservation in the limit $m_{b} \rightarrow 0$, as is reflected in Eq. (7). Using Eq. (6), we have

$$
\begin{gathered}
\hat{\Gamma}_{b, \mathrm{LO}}^{\text {unpol }}=F(1+2 \omega)(1-\omega)^{2}, \\
\hat{\Gamma}_{b, \mathrm{LO}}^{\text {pol }}=F(1-2 \omega)(1-\omega)^{2} .
\end{gathered}
$$

Inserting Eq. (7) in Eq. (2), we obtain a very simple formula for the final $\mathrm{LO}$ result:

$$
\begin{aligned}
& \frac{d^{3} \Gamma_{\mathrm{LO}}}{d x_{B} d \cos \theta d \cos \theta_{P}} \\
& =\frac{3}{8} F(1-\omega)^{2} D_{b}\left(x_{B}, \mu_{F}\right)\left[\sin ^{2} \theta\left(1+P \cos \theta_{P}\right)\right. \\
& \left.\quad+\omega(1-\cos \theta)^{2}\left(1-P \cos \theta_{P}\right)\right] .
\end{aligned}
$$

The Feynman diagrams contributing to the partial decay width of the process in Eq. (1) at NLO are depicted in Fig. 2. The NLO coefficient functions of the unpolarized case may be found in Appendix A of Ref. [8] and those of the polarized case are presented in the Appendix of this 

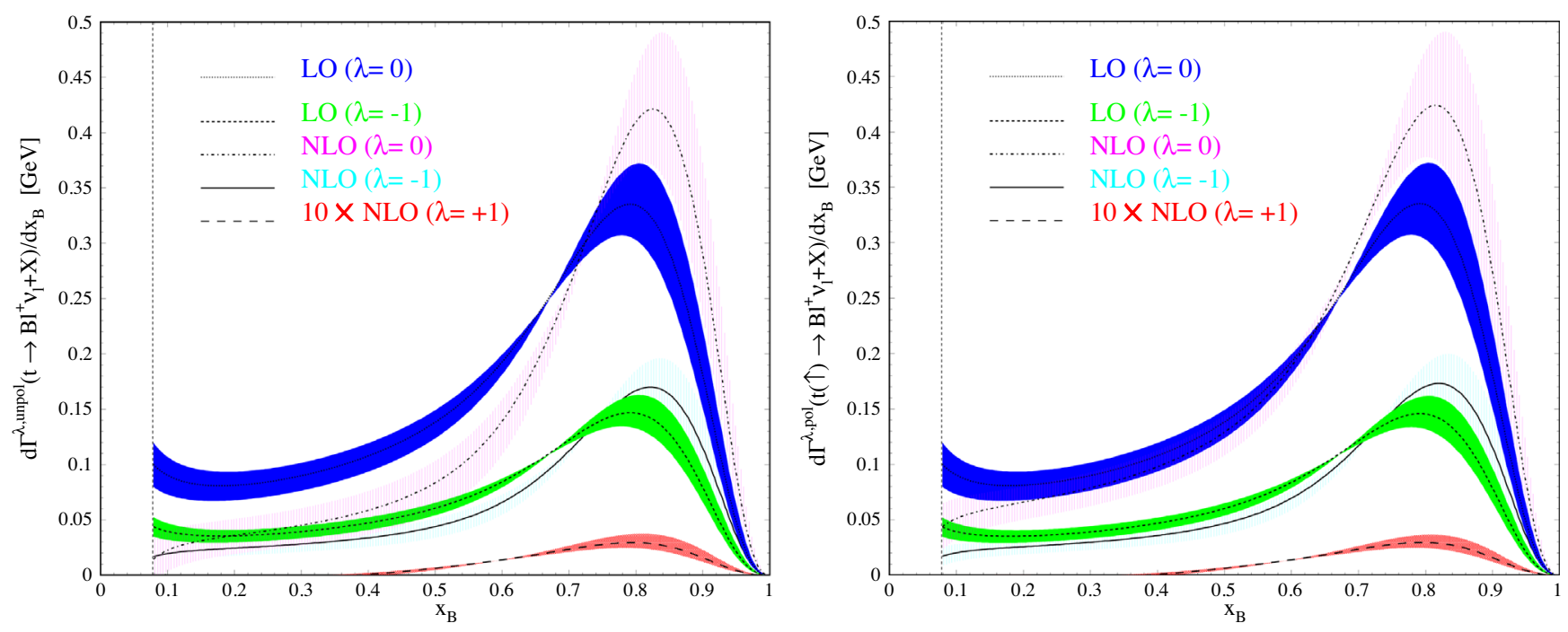

FIG. 3. LO and NLO results for (a) $d \Gamma^{\lambda, \text { unpol }} / d x_{B}$ and (b) $d \Gamma^{\lambda, \text { pol }} / d x_{B}$ with $\lambda=0, \pm 1$ as functions of $x_{B} . d \Gamma_{\mathrm{NLO}}^{+, \text {unpol }} / d x_{B}$ and $d \Gamma_{\mathrm{NLO}}^{+, \text {pol }} / d x_{B}$ are rescaled by a factor of 10 for better visibility. The theoretical uncertainties are indicated by the shaded bands.

paper. At NLO, the case $\lambda=+1$ is enabled by the presence of the additional spin-one gluon even for $m_{b}=0$.

At this point, we compare our new analytic results with the literature. In Refs. [25,26], process (1) was also considered at NLO using the narrow-width approximation and putting $m_{b}=0$, but treating the final state in a less differential fashion, which does not allow for the convolution with $a \rightarrow B$ FFs on the basis of the ZM-VFNS. We can compare our results for $\hat{\Gamma}_{b}^{\lambda, \text { pol }}$ with Refs. [25,26] upon integration over $x_{b}$ in the range $0 \leq x_{b} \leq 1$. Specifically, the quantities $\hat{\Gamma}_{U}^{P}, \hat{\Gamma}_{L}^{P}$, and $\hat{\Gamma}_{F}^{P}$ listed in Eqs. (18)-(20) of Ref. [25] (see also Eqs. (42)-(44) in Ref. [26]) are related to $\hat{\Gamma}_{b}^{\lambda, \text { pol }}$ as $\hat{\Gamma}_{b}^{0, \text { pol }} / \hat{\Gamma}_{b, \mathrm{LO}}^{\text {unpol }}=\hat{\Gamma}_{L}^{P}$ and $\hat{\Gamma}_{b}^{ \pm \text {,pol }} / \hat{\Gamma}_{b, \mathrm{LO}}^{\text {unpol }}=\left(\hat{\Gamma}_{U}^{P} \pm \hat{\Gamma}_{F}^{P}\right) / 2$. With these identifications, we fully agree with Refs. [25,26].

\section{NUMERICAL RESULTS}

We are now in a position to explore the phenomenological consequences of our results by performing a numerical analysis. We adopt from Ref. [1] the input parameter values $G_{F}=1.1663787 \times 10^{-5} \mathrm{GeV}^{-2}, m_{W}=$ $80.379 \mathrm{GeV}, m_{t}=172.4 \mathrm{GeV}, m_{B}=5.279 \mathrm{GeV},\left|V_{t b}\right|=$ 1 , and $B\left(W^{+} \rightarrow \ell^{+} \nu_{\ell}\right)=10.86 \%$. We evaluate $\alpha_{s}^{\left(n_{f}\right)}\left(\mu_{R}\right)$ at NLO (NNLO) in the $\overline{\mathrm{MS}}$ scheme using Eq. (4) of Ref. [31], retaining only the first two (three) terms on the right-hand side, with $n_{f}=5$ active quark flavors and asymptotic scale parameter $\Lambda_{\overline{\mathrm{MS}}}^{(5)}=225 \mathrm{MeV}$ $(207 \mathrm{MeV})$ adjusted such that $\alpha_{s}^{(5)}\left(m_{Z}\right)=0.1179$ for $m_{Z}=$ $91.1876 \mathrm{GeV}$ [1]. This yields $\alpha_{s}^{(5)}\left(m_{t}\right)=0.1076(0.1076)$. As already mentioned in Sec. I, we use the up-to-date $B$ FFs from Ref. [23], both at NLO and NNLO. For definiteness, we identify $\mu_{R}=\mu_{F}=\xi m_{t}$ and vary $\xi$ from $1 / 2$ to 2 about the default value 1 to estimate the theoretical uncertainty due to the lack of knowledge of higher-order corrections.

The angular dependencies at the parton level, in Eqs. (3) and (5), are passed on to the hadron level via Eq. (2). The hadron level counterparts, $d \Gamma^{\lambda} / d x_{B}$, of the coefficient functions $d \hat{\Gamma}_{a}^{\lambda} / d x_{a}$ in Eq. (5) may be projected out from the measured $\theta$ distribution $d \Gamma /\left(d x_{B} d \cos \theta\right)$ as explained in Sec. 4 of Ref. [8] (see Eqs. (21)-(23) therein) and thus represent physical observables by themselves. In Ref. [8], the top quarks were taken to be unpolarized, i.e., according to our present notation, $d \Gamma^{\lambda \text {,unpol }} / d x_{B}$ were considered. In the following, we complement the study of Ref. [8] by presenting predictions for $d \Gamma^{\lambda, p o l} / d x_{B}$. For the sake of a coherent treatment, we also provide the analogous predictions for $d \Gamma^{\lambda, \text { unpol }} / d x_{B}$, thus updating the analysis of Ref. [8].

Our central predictions are of NLO. To assess their significance, we compare them with the respective LO results. Our LO predictions are slightly inconsistent because they are evaluated with NLO FFs. Unfortunately, Ref. [23] does not provide a LO FF set. By the way, the same is true for Ref. [16], to which we could have resorted otherwise. On the other hand, Ref. [23] also supplies a NNLO set. While consistent NNLO predictions are unfeasible in the absence of NNLO parton-level results, this still offers us the opportunity to get a first impression of the typical magnitude of the NNLO effects. In our pseudo-NNLO analysis, besides using NNLO FFs, we also evaluate $\alpha_{s}\left(\mu_{R}\right)$ at NNLO as explained above.

In Fig. 3, we study the $x_{B}$ dependencies of $d \Gamma^{\lambda \text {,unpol }} / d x_{B}$ and $d \Gamma^{\lambda, \mathrm{pol}} / d x_{B}$ for $\lambda=0, \pm 1$ at NLO and compare them with the respective LO results for $\lambda=0,-1$. As explained in Sec. II, $d \Gamma^{+, \text {unpol }} / d x_{B}$ and $d \Gamma^{+, \text {pol }} / d x_{B}$ vanish at LO in our approximation. The theoretical uncertainties are 

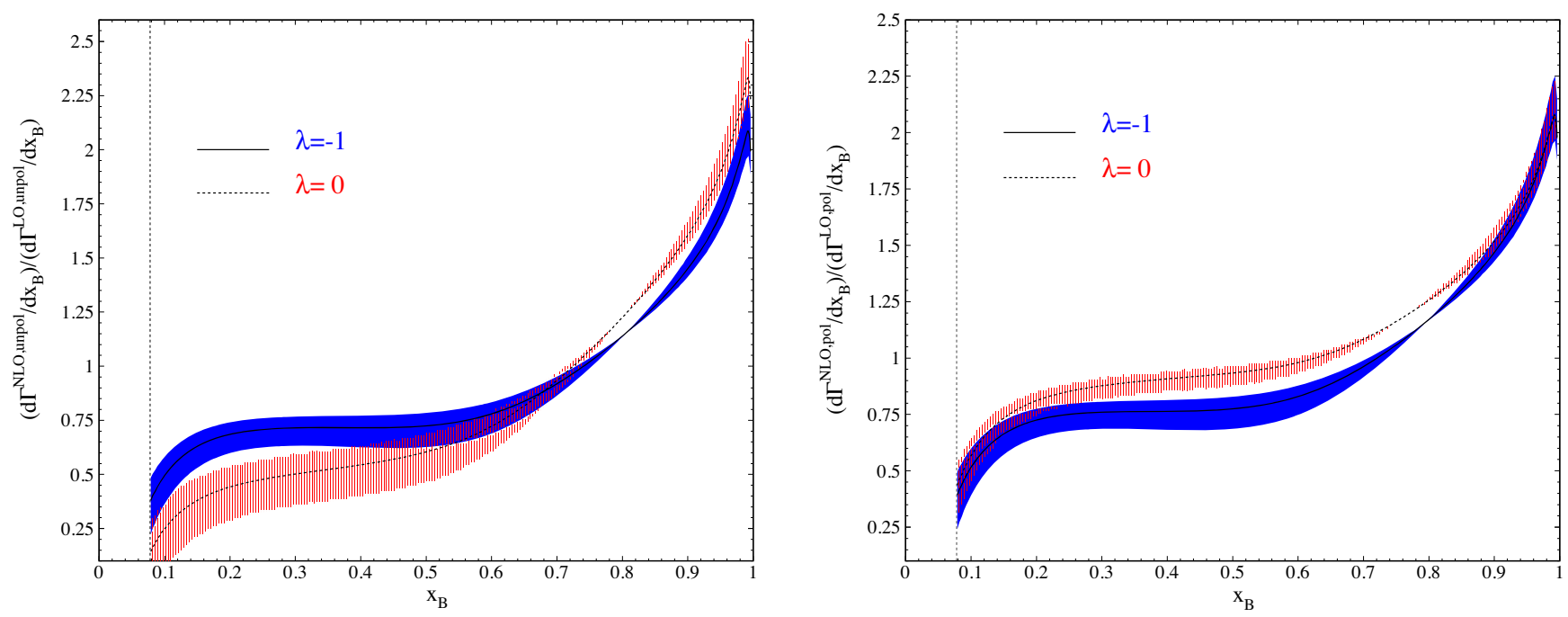

FIG. 4. NLO results for (a) $d \Gamma^{\lambda \text {,unpol }} / d x_{B}$ and (b) $d \Gamma^{\lambda \text {,pol }} / d x_{B}$ with $\lambda=0,-1$, normalized to the respective default LO results, as functions of $x_{B}$. The theoretical uncertainties of the NLO results are indicated by the shaded bands.

indicated by the shaded bands. The slight inconsistency in our LO analysis mentioned above, not only affects the default predictions, but also the estimation of the theoretical uncertainty, which is expected to be slightly larger if the $\mu_{F}$ dependence is subject to LO DGLAP evolution. Comparing Figs. 3(a) and 3(b), we observe that $d \Gamma^{\lambda, \text { unpol }} / d x_{B}$ and $d \Gamma^{\lambda, \text { pol }} / d x_{B}$ are very similar as for normalization and line shape. Each $x_{B}$ distribution exhibits a maximum close to $x_{B}=0.8$. Longitudinal $W$-boson helicity is favored, with $d \Gamma_{\mathrm{LO} / \mathrm{NLO}}^{0, \mathrm{unol} / \mathrm{pol}} / d x_{B}$ being more than twice as large as $d \Gamma_{\mathrm{LO} / \mathrm{NLO}}^{-, \mathrm{unol} / \mathrm{pol}} / d x_{B}$ for negative $W$-boson helicity $\lambda=-1$. On the other hand, positive $W$-boson helicity $\lambda=+1$ is perturbatively suppressed; rescaling $d \Gamma_{\mathrm{NLO}}^{+, \text {unpol} / \mathrm{pol}} / d x_{B}$ by the inverse couplant, $2 \pi / \alpha_{s}^{(5)}\left(m_{t}\right)$,

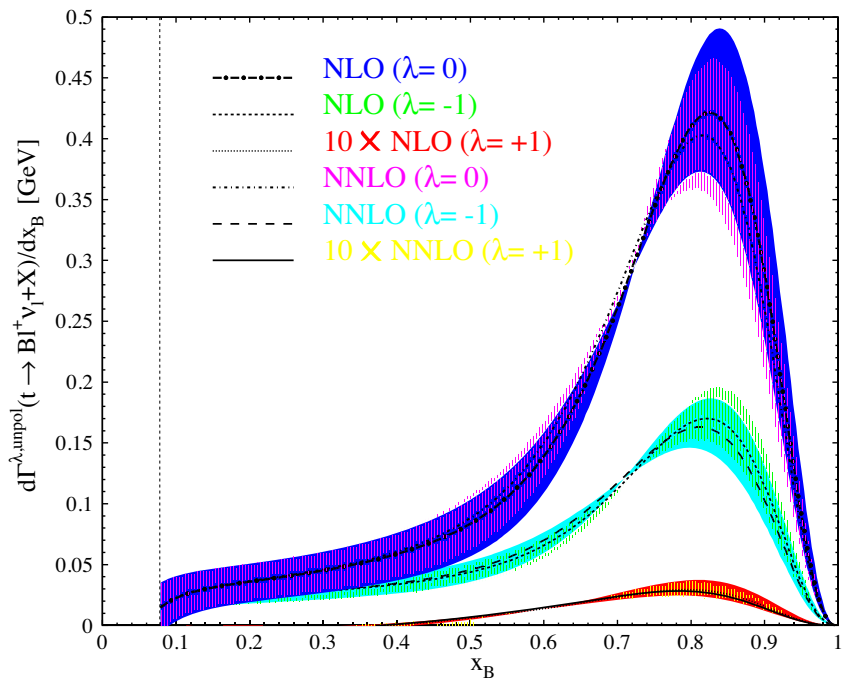

brings it up to the level of $d \Gamma_{\mathrm{NLO}}^{- \text {,unpol/pol }} / d x_{B}$. The NLO corrections have a significant effect on the $x_{B}$ distributions for $\lambda=0,-1$, by raising their peaks and lowering their small- $x_{B}$ tails. To render these features more visible, we present, in Fig. 4, the QCD correction $(K)$ factors for $\lambda=0$, -1 , which we evaluate by normalizing the NLO predictions including their theoretical-uncertainty bands relative to the default LO predictions. From Figs. 4(a) and 4(b), we observe that, both for unpolarized and polarized top quarks, the $K$ factors steadily increase by one order of magnitude, typically from 0.2 to 2 , as $x_{B}$ runs across its range of values. We conclude from Figs. 3 and 4 that the NLO corrections are quite significant and should be taken into account in theoretical interpretations of future top-quark polarization measurements.

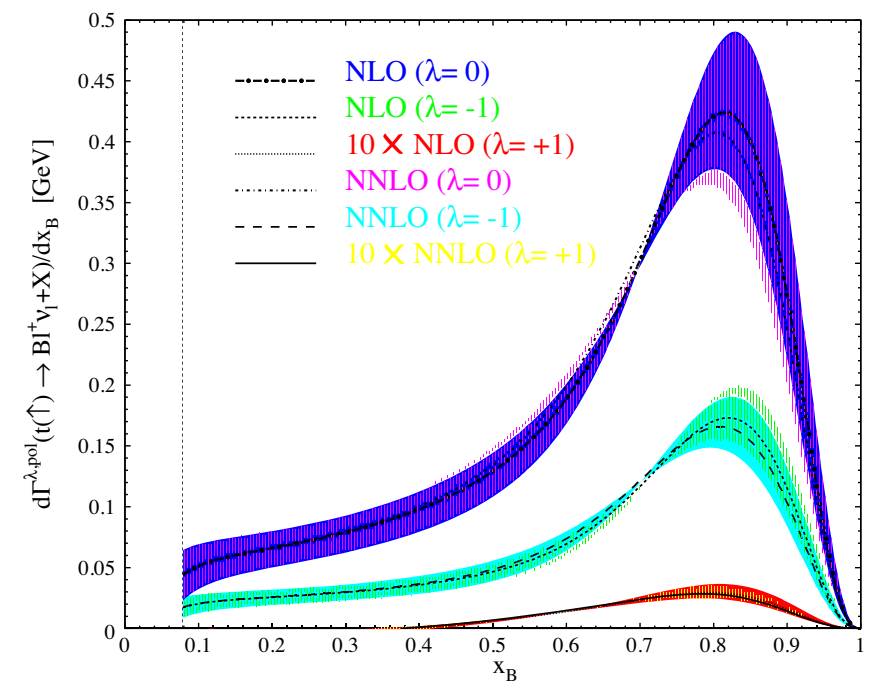

FIG. 5. NLO and pseudo-NNLO results for (a) $d \Gamma^{\lambda, \text { unpol }} / d x_{B}$ and (b) $d \Gamma^{\lambda, \text { pol }} / d x_{B}$ with $\lambda=0, \pm 1$ as functions of $x_{B}$. $d \Gamma_{\mathrm{NLO} / \mathrm{NNLO}}^{+, \text {unpol }} / d x_{B}$ and $d \Gamma_{\mathrm{NLO} / \mathrm{NNLO}}^{+, \text {pol }} / d x_{B}$ are rescaled by a factor of 10 for better visibility. The theoretical uncertainties are indicated by the shaded bands. 

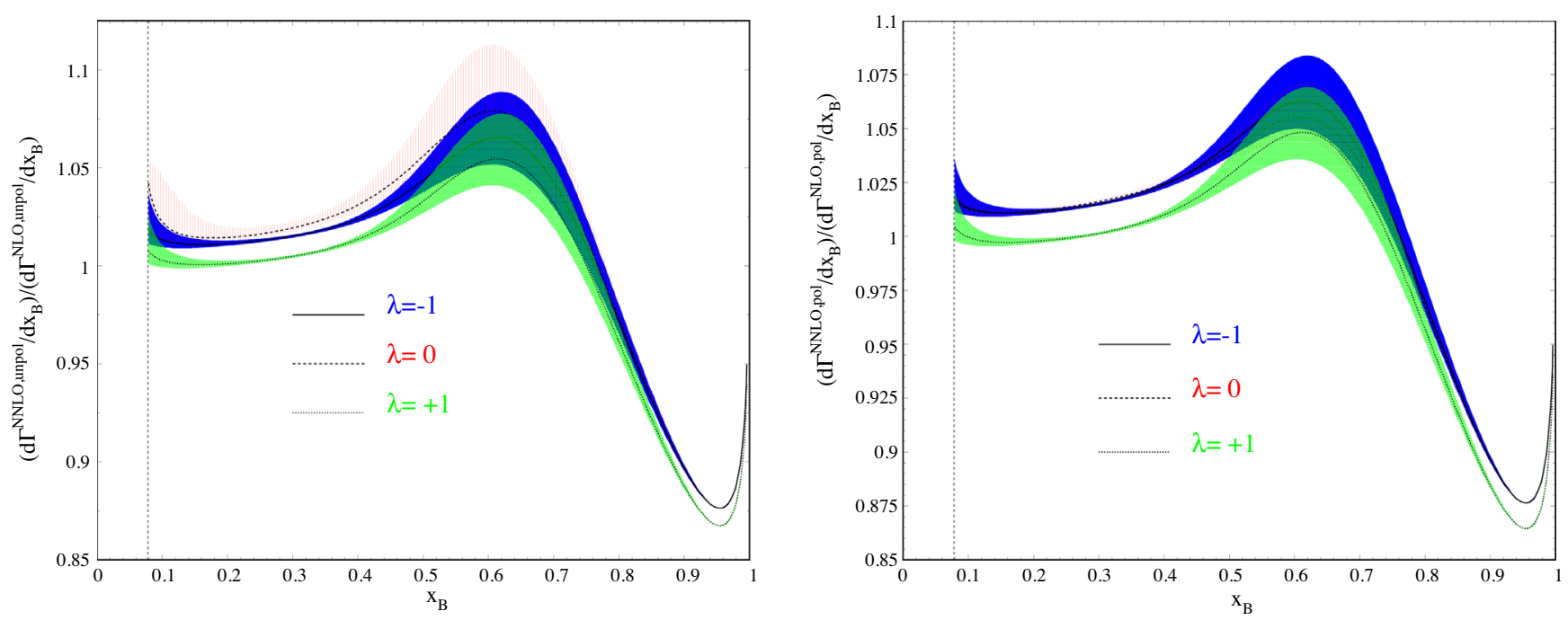

FIG. 6. Pseudo-NNLO results for (a) $d \Gamma^{\lambda \text {,unpol }} / d x_{B}$ and (b) $d \Gamma^{\lambda, \text { pol }} / d x_{B}$ with $\lambda=0, \pm 1$, normalized to the respective default NLO results, as functions of $x_{B}$. The theoretical uncertainties of the pseudo-NNLO results are indicated by the shaded bands.

In Fig. 5, compare our pseudo-NNLO evaluations of $d \Gamma^{\lambda, \text { unpol }} / d x_{B}$ and $d \Gamma^{\lambda, \text { pol }} / d x_{B}$ for $\lambda=0, \pm 1$ with the respective NLO results already presented in Fig. 3. For better visibility, we also plot, in Fig. 6, the ratios of the pseudo-NNLO results including their theoreticaluncertainty bands and the default NLO predictions. We conclude from Figs. 5 and 6 that the NNLO effects are likely to be relatively modest, of the order of $10 \%$ or less.

\section{SUMMARY AND CONCLUSIONS}

We studied the inclusive production of bottom-flavored hadrons from semileptonic decays of polarized top quarks at NLO in the ZM-FVNS using the narrow-width approximation for the intermediate $W$ bosons, whose helicities we distinguished. Specifically, we considered the partial decay width differential in the scaled bottom-hadron energy $x_{B}$, the azimuthal angle $\theta$ of the charged lepton in the $W$-boson rest frame, and the azimuthal angle $\theta_{P}$ of the top-quark polarization in the top-quark rest frame. In our numerical analysis, we employed up-to-date $B$ FFs, recently determined from a global fit to all available $e^{+} e^{-}$data [23]. We thus extended our previous study in Ref. [8], which was restricted to unpolarized top quarks. In Ref. [8], we had convinced ourselves, by comparing evaluations in the GMVFNS and ZM-VFNS, that finite- $m_{b}$ corrections may be safely neglected. On the other hand, we retained finite- $m_{B}$ corrections, which reduce the available phase space and lead to visible effects at small values of $x_{B}$. We provided full analytic results, ready to be used by the interested reader.

We found the NLO QCD corrections to be quite significant, inducing a reduction in the lower $x_{B}$ range and an enhancement in the upper $x_{B}$ range, with $K$ factors ranging from 0.2 to 2 . On the other hand, including partial information from NNLO, contained in the evaluation of the $B$ FFs and $\alpha_{s}\left(\mu_{R}\right)$, turned out to yield only mild modifications, with the due caveat that such results suffer from a violation of renormalization group invariance already in the considered order.

Our combined results, from Ref. [8] and this paper, help us to fill an important gap in the theoretical interpretation of recent measurements of the top-quark polarization and the $t \bar{t}$ spin correlations in dilepton final states at the LHC [2,3]. In particular, it will be interesting to see if these theoretical improvements will contribute to reconciling the ATLAS [2] and CMS [3] measurements of $A_{\left|\Delta \phi_{\ell \ell}\right|}$ with the SM expectations.

\section{ACKNOWLEDGMENTS}

We thank G. Kramer for useful discussions at the initial stage of this work and C. Schwanenberger for detailed information on Ref. [3]. The work of B. A. K. was supported in part by the German Federal Ministry for Education and Research BMBF through Grant No. 05H18GUCC1. The work of S. M. M. N. was supported in part by the Iran National Science Foundation INSF through Grant No. 97005414.

\section{APPENDIX}

In this Appendix, we list the coefficient functions $d \hat{\Gamma}_{a}^{\lambda, \text { pol }} / d x_{a}$ appearing in Eq. (5) at NLO in the ZM-VFNS with $m_{b}=0$. They possess the following structure: 


$$
\begin{aligned}
& \frac{1}{\hat{\Gamma}_{b, \mathrm{LO}}^{\mathrm{pol}}} \frac{d \hat{\Gamma}_{a}^{0, \mathrm{pol}}}{d x_{a}}=\frac{1}{1-2 \omega}\left[\delta_{a b} \delta\left(1-x_{a}\right)+\frac{\alpha_{s}\left(\mu_{R}\right)}{2 \pi}\left(P_{a b}\left(x_{a}\right) \ln \frac{m_{t}^{2}}{\mu_{F}^{2}}+C_{F} C_{a}^{0, \mathrm{pol}}\left(x_{a}\right)\right)\right] \\
& \frac{1}{\hat{\Gamma}_{b, \mathrm{LO}}^{\mathrm{pol}}} \frac{d \hat{\Gamma}_{a}^{-, \mathrm{pol}}}{d x_{a}}=\frac{-2 \omega}{1-2 \omega}\left[\delta_{a b} \delta\left(1-x_{a}\right)+\frac{\alpha_{s}\left(\mu_{R}\right)}{2 \pi}\left(P_{a b}\left(x_{a}\right) \ln \frac{m_{t}^{2}}{\mu_{F}^{2}}+C_{F} C_{a}^{-, \mathrm{pol}}\left(x_{a}\right)\right)\right] \\
& \frac{1}{\hat{\Gamma}_{b, \mathrm{LO}}^{\mathrm{pol}}} \frac{d \hat{\Gamma}_{a}^{+, \mathrm{pol}}}{d x_{a}}=\frac{4 \omega}{(1-2 \omega)(1-\omega)^{2}} \frac{\alpha_{s}\left(\mu_{R}\right)}{2 \pi} C_{F} C_{a}^{+, \mathrm{pol}}\left(x_{a}\right),
\end{aligned}
$$

where

$$
\begin{aligned}
& P_{q q}(x)=C_{F}\left(\frac{1+x^{2}}{1-x}\right)_{+}, \\
& P_{g q}(x)=C_{F} \frac{1+(1-x)^{2}}{x}
\end{aligned}
$$

are the timelike $q \rightarrow q$ and $q \rightarrow g$ splitting functions at LO.

For $a=b$, we have

$$
\begin{aligned}
& C_{b}^{0, p o l}(x)=-\delta(1-x)\left[2 \ln \omega \ln (1-\omega)+4 \operatorname{Li}_{2}(\omega)+\frac{2 \omega}{1-\omega} \ln \omega+6\right]+2\left(1+x^{2}\right)\left(\frac{\ln (1-x)}{1-x}\right)_{+}+2 \frac{1+x^{2}}{(1-x)_{+}} \ln (x(1-\omega)) \\
& +\frac{8(x-2)(1-x)^{2}}{x^{2}\left(S x^{2}-2 x+2\right)}+2 \omega(1-x)-\frac{12}{x}+\frac{8}{x^{2}}+\frac{6}{1-x}-\frac{(1+x)^{2}}{1-x}-\frac{1+x^{2}}{1-x} R_{1} \\
& +\left(1-\left|1-2 x+2 S x^{2}\right|\right)\left(6 \frac{1-x}{x^{2}}-\frac{1}{(1-x)(1-\omega)}+6 \frac{(1-x)^{2}(x-2)^{2}}{x^{2}\left(S x^{2}-2 x+2\right)^{2}}\right. \\
& \left.+\frac{1}{2 x^{2}\left(S x^{2}-2 x+2\right)}\left[9 x^{3}-57 x^{2}+97 x-\frac{1}{1-x}-47\right]\right) \\
& +\frac{R_{2}}{S^{3 / 2}\left(S x^{2}-2 x+2\right)^{5 / 2}}\left(x^{3} S^{4}\left(-x^{3}+7 x^{2}-22 x+10+\frac{2}{1-x}\right)+x S^{3}\left(x^{4}+4 x^{3}+25 x^{2}-32 x+12\right)\right. \\
& \left.-2(1-x)^{2}+S^{2}(1-x)\left(5 x^{3}+21 x^{2}-12 x+4\right)-S(1-x)\left(9 x^{2}-4 x-4\right)\right), \\
& C_{b}^{-, \mathrm{pol}}(x)=-\delta(1-x)\left[2 \ln \omega \ln (1-\omega)+4 \mathrm{Li}_{2}(\omega)+\frac{2 \omega}{1-\omega} \ln \omega+6+\frac{1-\omega}{\omega} \ln (1-\omega)\right]+2\left(1+x^{2}\right)\left(\frac{\ln (1-x)}{1-x}\right)_{+} \\
& +2 \frac{1+x^{2}}{(1-x)_{+}} \ln (x(1-\omega))-\frac{1+x^{2}}{2(1-x)} R_{1}+\frac{(1-S)^{2}}{\omega S}+\frac{\omega+S x}{\omega}-\frac{\omega}{2 S(1-x)}+\frac{(1-S-S x)}{S} \ln (1-2 S x) \\
& -\frac{3 S(2 x-1)+6(1-x)}{2 S\left(S x^{2}-2 x+2\right)^{2}}+\frac{S^{2}(1+3 x)+4 x \omega-3 S}{2 \omega S\left(S x^{2}-2 x+2\right)}+B_{3}\left|1-2 x+2 S x^{2}\right|+B_{1} \ln \left|2 S x^{2}-2 x+1\right| \\
& +\frac{R_{3}}{S^{2} \sqrt{\omega}} B_{2}-\frac{R_{2}}{2 \sqrt{S x^{2}-2 x+2}}\left(x^{2} \sqrt{S}+\frac{(S-1) x}{\sqrt{S}}-\frac{2 \sqrt{S}}{1-x}+\frac{2 S^{2}-5 S+7}{S^{\frac{3}{2}}}\right. \\
& \left.+\frac{S x\left(4 S^{2}-23 S+16\right)-S(28-19 S)+6}{S^{\frac{5}{2}}\left(S x^{2}-2 x+2\right)}-\frac{3 S x\left(12-9 S+S^{2}\right)+15 S(S-2)+12(1-x)}{S^{\frac{5}{2}}\left(S x^{2}-2 x+2\right)^{2}}\right),
\end{aligned}
$$




$$
\begin{aligned}
C_{b}^{+, \mathrm{pol}}(x)= & \frac{\left(1+x^{2}\right) R_{1}}{1-x} S^{2}-\frac{2(1+x) S^{3}}{\omega}+2 S[1+S x+(1-S-S x) \ln (1-2 S x)] \\
& -\frac{(17-21 x) S^{3}+(20 x-27) S^{2}+4(2-x) S}{\omega\left(S x^{2}-2 x+2\right)}+\frac{6(1-x) S-3(1-2 x) S^{2}}{\left(S x^{2}-2 x+2\right)^{2}}+\frac{\omega S}{1-x}+\frac{2 R_{3}}{\sqrt{\omega}} B_{2} \\
& -2 S^{2} B_{3}\left|1-2 x+2 S x^{2}\right|+2 S^{2} B_{1} \ln \left|2 S x^{2}-2 x+1\right|-\frac{R_{2}(1-x) \sqrt{S}}{x^{3} \sqrt{S x^{2}-2 x+2}}\left(11+7 x^{2}-16 x+\frac{6(2-x)(1-x)^{3}}{\left(2-2 x+S x^{2}\right)^{2}}\right. \\
& \left.+\frac{16 x^{3}-58 x^{2}+69 x-28}{2-2 x+S x^{2}}+\frac{S x^{2}\left(x^{2}+5 x-4\right)}{1-x}+\frac{S^{2} x^{4}\left(1+x^{2}\right)}{(1-x)^{2}}\right)
\end{aligned}
$$

where $S=(1-\omega) / 2$,

$$
\begin{aligned}
& R_{1}=\ln \left[(1-S) x^{2}-x+\frac{1}{2}+\frac{1}{2}\left|2 S x^{2}-2 x+1\right|\right], \\
& R_{2}=\ln \left[(1-x)(1-3 S x)+S x^{2}(1-2 S x)+\sqrt{S\left(S x^{2}-2 x+2\right)}\left|2 S x^{2}-2 x+1\right|\right]-\ln \left[1+(S-1) x+\sqrt{S\left(S x^{2}-2 x+2\right)}\right], \\
& R_{3}=\ln [1-x(1-\sqrt{\omega})]-\ln |1-x(1+\sqrt{\omega})|, \\
& B_{1}=x-\frac{1-S}{S}-\frac{1-x}{2 S\left(2-2 x+S x^{2}\right)^{2}}\left[5 S^{2} x^{2}-2(1-x)-2 S\left(x^{2}+3 x-3\right)\right] \\
& B_{2}=-\omega S+x S^{2}+\frac{S x\left(-4 S^{2}+7 S-2\right)+S(8-10 S)-2}{2\left(S x^{2}-2 x+2\right)}-\frac{\omega\left(S^{2} x-4 S x+3 S+2 x-2\right)}{\left(S x^{2}-2 x+2\right)^{2}} \\
& B_{3}=\frac{1}{2 S(1-x)}+\frac{1}{1-2 S x}+\frac{6(1-x)-3 S(1-2 x)}{2 S\left(S x^{2}-2 x+2\right)^{2}}+\frac{S^{2}(3 x-5)+S(11-2 x)-4}{2 \omega S\left(S x^{2}-2 x+2\right)}
\end{aligned}
$$

For $a=g$, we have

$$
\begin{aligned}
C_{g}^{0, \mathrm{pol}}(x)= & \frac{1+(1-x)^{2}}{x}\left(-R_{1}+2 \ln [x(1-\omega)(1-x)]\right)+\left(8 S-x-26+\frac{13}{S}+\frac{26 S^{2}-38 S+13}{2 S^{3} x^{2}}+\frac{-44 S^{2}+88 S-35}{2 x S^{2}}\right) R_{4} \\
& +\left(\frac{1-6 S}{2(2 S x-1)}+9 \frac{1-S}{2 S x}+\frac{-4 S^{2}+25 S-13}{2 S^{2} x^{2}}+\frac{\omega}{2(1-2 S x)^{2}}\right)\left|2 S x^{2}-2 x+1\right| \\
& +2(7-6 S)+(1-4 S) x+7 \frac{7 S-5}{2 S x}+\frac{4 S^{2}-25 S+13}{2 S^{2} x^{2}}, \\
C_{g}^{-, \mathrm{pol}}(x)= & \frac{1+(1-x)^{2}}{2 x}\left(4 \ln [x(1-\omega)(1-x)]-R_{1}\right)+\frac{1-6 S}{4 \omega} x-\frac{\omega^{2}}{32 S^{3} x^{2}(1-2 S x)^{2}}-\frac{(10 S-7) \omega}{32 S^{3} x^{2}(1-2 S x)} \\
& +\frac{1+5 S-8 S^{2}}{2 S \omega}-A_{3} R_{4}+\frac{\left|2 S x^{2}-2 x+1\right|}{8 \omega S^{2} x^{2}} A_{5}+\frac{A_{2}}{2 S^{3} x^{2}} \ln (1-2 S x)+\frac{R_{3} A_{4}}{2 \sqrt{\omega} S^{3} x^{2}}-A_{1} \frac{\ln \left|2 S x^{2}-2 x+1\right|}{2 x^{2} S^{3}} \\
& +\frac{1}{16 \omega x S^{2}}\left(48 S^{3}+72 S^{2}-50 S-5\right)+\frac{1}{16 \omega S^{3} x^{2}}\left(32 S^{3}-88 S^{2}+44 S-3\right), \\
C_{g}^{+, \mathrm{pol}}(x)= & \frac{1}{2 S x^{2}}\left[\frac{2 R_{3}}{\sqrt{\omega}} A_{4}+2 x\left(1+(1-x)^{2}\right) S^{3} R_{1}+4 S^{3} x^{2} A_{3} R_{4}+2 A_{2} \ln (1-2 S x)-2 A_{1} \ln \left|2 S x^{2}-2 x+1\right|\right. \\
& -\frac{S A_{5}}{2 \omega}\left|2 S x^{2}-2 x+1\right|+\frac{S}{4 \omega}\left(4 S^{2}(1+2 S) x^{3}+8 S\left(4 S^{2}-7 S+1\right) x^{2}+\frac{8 \omega^{2} x}{S(1-4 S)}-\left(96 S^{2}-70 S+5\right) x\right. \\
& \left.\left.+\frac{32 S^{2}-12 S-3}{S}-\frac{40 S^{3}-68 S^{2}+38 S-7}{2 S(1-2 S x)}+\frac{8 S^{3}-12 S^{2}+6 S-1}{2 S(1-2 S x)^{2}}\right)\right],
\end{aligned}
$$

where 


$$
\begin{aligned}
& R_{4}=\ln \left[1-S\left(-2 S x^{2}+2 x+1-\left|2 S x^{2}-2 x+1\right|\right)\right]-\ln [1-2 S x], \\
& A_{1}=2 x^{2}(x-1) S^{3}+\left(-2 x^{2}+5 x+1\right) S^{2}-2(2+x) S+2, \\
& A_{2}=x\left(x^{2}-2\right) S^{3}+\left(-2 x^{2}+5 x+1\right) S^{2}-2(2+x) S+2, \\
& A_{3}=\frac{2+x^{2}}{2 x}-\frac{5 x^{2}+5 x+1}{2 S x^{2}}-\frac{7-S(8+15 x)}{4 x^{2} S^{3}}, \\
& A_{4}=2 x\left(2-x^{2}\right) S^{3}+\left(2 x^{2}-7 x-4\right) S^{2}+2(x+3) S-2, \\
& A_{5}=20 S-11-S x(10 S-7)-\frac{\omega(7-10 S)}{2(1-2 S x)}+\frac{\omega^{2}}{2(1-2 S x)^{2}} .
\end{aligned}
$$

Adding Eqs. (A3)-(A5) for $a=b$ and Eqs. (A7)-(A9) for $a=g$ according to Eq. (6), we obtain the respective coefficient functions pertaining to the case where $\theta$ is integrated over. Specifically, we have

$$
\begin{aligned}
& \frac{1}{\hat{\Gamma}_{b, \mathrm{LO}}^{\mathrm{pol}}} \frac{d \hat{\Gamma}_{b}^{\mathrm{pol}}}{d x_{b}}=\delta\left(1-x_{b}\right)+\frac{\alpha_{s}\left(\mu_{R}\right)}{2 \pi}\left(P_{q q}\left(x_{b}\right) \ln \frac{m_{t}^{2}}{\mu_{F}^{2}}+C_{F} C_{b}^{\mathrm{pol}}\left(x_{b}\right)\right), \\
& \frac{1}{\hat{\Gamma}_{b, \mathrm{LO}}^{\mathrm{pol}}} \frac{d \hat{\Gamma}_{g}^{\mathrm{pol}}}{d x_{g}}=\frac{\alpha_{s}\left(\mu_{R}\right)}{2 \pi}\left(P_{g q}\left(x_{g}\right) \ln \frac{m_{t}^{2}}{\mu_{F}^{2}}+C_{F} C_{g}^{\mathrm{pol}}\left(x_{g}\right)\right),
\end{aligned}
$$

where

$$
\begin{aligned}
C_{b}^{\mathrm{pol}}(x)= & \delta(1-x)\left[-2 \ln \omega \ln (1-\omega)+\frac{2(1-\omega)}{1-2 \omega} \ln (1-\omega)-4 \mathrm{Li}_{2}(\omega)-\frac{2 \omega}{1-\omega} \ln \omega-6\right] \\
& +2\left(1+x^{2}\right)\left(\frac{\ln (1-x)}{1-x}\right)_{+}+2 \frac{1+x^{2}}{(1-x)_{+}} \ln (x(1-\omega))+\frac{1-S}{S\left(S x^{2}-2 x+2\right)}-\frac{2}{1-4 S}-\frac{\omega}{S(1-x)}-1-x \\
& -\frac{1+x^{2}}{1-x} R_{1}+\left(\frac{1}{S(1-x)}+\frac{4 \omega}{(1-4 S)(1-2 S x)}-\frac{1-S}{S\left(S x^{2}-2 x+2\right)}\right)\left|2 S x^{2}-2 x+1\right| \\
& -\frac{R_{2}}{\sqrt{S\left(S x^{2}-2 x+2\right)}}\left(\frac{1}{S}+\frac{2}{1-4 S}+\frac{\left(13 S-5-4 S^{2}\right) x}{1-4 S}-\frac{S x\left(x^{2}-x+2\right)}{1-x}-\frac{(1-S)[1-(1-S) x]}{S\left(S x^{2}-2 x+2\right)}\right), \\
C_{g}^{\mathrm{pol}}(x)= & \frac{1+(1-x)^{2}}{x}\left(-R_{1}+2 \ln [x(1-\omega)(1-x)]\right)-\left(x-\frac{1+\omega^{2}}{4 S^{3} x^{2}}-\frac{8 S^{2}-6 S+3}{S(4 S-1)}-\frac{2-\omega^{2}(2 \omega-5)}{2 S^{2}(1-4 S) x}\right) R_{4} \\
& +\left(\frac{S-1}{2 S^{2} x^{2}}+\frac{\omega}{2(1-2 S x)^{2}}-\frac{12 S^{2}-15 S+7}{2 S(1-4 S) x}-\frac{24 S^{2}-26 S+9}{2(1-4 S)(1-2 S x)}\right)\left|2 S x^{2}-2 x+1\right|+x+\frac{1-S}{2 S^{2} x^{2}} \\
& -\frac{2}{1-4 S}+\frac{12 S^{2}-7 S+5}{2 S(1-4 S) x} .
\end{aligned}
$$

[1] P. A. Zyla et al. (Particle Data Group), Review of particle physics, Prog. Theor. Exp. Phys. 2020, 083C01 (2020).

[2] M. Aaboud et al. (ATLAS Collaboration), Measurements of top-quark pair spin correlations in the $e \mu$ channel at $\sqrt{s}=13 \mathrm{TeV}$ using $p p$ collisions in the ATLAS detector, Eur. Phys. J. C 80, 754 (2020).
[3] A. M. Sirunyan et al. (CMS Collaboration), Measurement of the top quark polarization and $\bar{t} \overline{\mathrm{t}}$ spin correlations using dilepton final states in proton-proton collisions at $\sqrt{s}=13$ TeV, Phys. Rev. D 100, 072002 (2019).

[4] W. Bernreuther and Z.-G. Si, Top quark spin correlations and polarization at the LHC: Standard model predictions 
and effects of anomalous top chromo moments, Phys. Lett. B 725, 115 (2013); 744, 413(E) (2015).

[5] W. Bernreuther, D. Heisler, and Z.-G. Si, A set of top quark spin correlation and polarization observables for the LHC: Standard model predictions and new physics contributions, J. High Energy Phys. 12 (2015) 026.

[6] P. Artoisenet, R. Frederix, O. Mattelaer, and R. Rietkerk, Automatic spin-entangled decays of heavy resonances in Monte Carlo simulations, J. High Energy Phys. 03 (2013) 015.

[7] J.C. Collins, Hard-scattering factorization with heavy quarks: A general treatment, Phys. Rev. D 58, 094002 (1998).

[8] B. A. Kniehl, G. Kramer, and S. M. Moosavi Nejad, Bottom-flavored hadrons from top-quark decay at nextto-leading order in the general-mass variable-flavor-number scheme, Nucl. Phys. B862, 720 (2012).

[9] T. Kneesch, B. A. Kniehl, G. Kramer, and I. Schienbein, Charmed-meson fragmentation functions with finite-mass corrections, Nucl. Phys. B799, 34 (2008).

[10] G. Kramer and H. Spiesberger, Inclusive $D^{*}$ production in photon-photon collisions at next-to-leading order QCD, Eur. Phys. J. C 22, 289 (2001).

[11] G. Kramer and H. Spiesberger, Inclusive photoproduction of $D^{*}$ mesons with massive charm quarks, Eur. Phys. J. C 38, 309 (2004).

[12] B. A. Kniehl, G. Kramer, I. Schienbein, and H. Spiesberger, Inclusive $D^{* \pm}$ production in $p \bar{p}$ collisions with massive charm quarks, Phys. Rev. D 71, 014018 (2005).

[13] B. A. Kniehl, G. Kramer, I. Schienbein, and H. Spiesberger, Collinear subtractions in hadroproduction of heavy quarks, Eur. Phys. J. C 41, 199 (2005).

[14] B. A. Kniehl, G. Kramer, I. Schienbein, and H. Spiesberger, Inclusive $B$-meson production at small $p_{T}$ in the generalmass variable-flavor-number scheme, Eur. Phys. J. C 75, 140 (2015).

[15] M. Benzke, M. V. Garzelli, B. A. Kniehl, G. Kramer, S. Moch, and G. Sigl, Prompt neutrinos from atmospheric charm in the general-mass variable-flavor-number scheme, J. High Energy Phys. 12 (2017) 021.

[16] B. A. Kniehl, G. Kramer, I. Schienbein, and H. Spiesberger, Finite-mass effects on inclusive $B$-meson hadroproduction, Phys. Rev. D 77, 014011 (2008).

[17] A. Heister et al. (ALEPH Collaboration), Study of the fragmentation of $b$ quarks into $B$ mesons at the $Z$ peak, Phys. Lett. B 512, 30 (2001).
[18] G. Abbiendi et al. (OPAL Collaboration), Inclusive analysis of the $b$ quark fragmentation function in $Z$ decays at LEP, Eur. Phys. J. C 29, 463 (2003).

[19] K. Abe et al. (SLD Collaboration), Precise Measurement of the $b$-Quark Fragmentation Function in $Z^{0}$ Boson Decays, Phys. Rev. Lett. 84, 4300 (2000).

[20] V. N. Gribov and L. N. Lipatov, Deep inelastic ep scattering in perturbation theory, Yad. Fiz. 15, 781 (1972) [Sov. J. Nucl. Phys. 15, 438 (1972)].

[21] G. Altarelli and G. Parisi, Asymptotic freedom in parton language, Nucl. Phys. B126, 298 (1977).

[22] Yu. L. Dokshitzer, Calculation of the structure functions for deep inelastic scattering and $e^{+} e^{-}$annihilation by perturbation theory in quantum chromodynamics, Zh. Eksp. Teor. Fiz. 73, 1216 (1977) [Sov. Phys. JETP 46, 641 (1977)].

[23] M. Salajegheh, S. M. Moosavi Nejad, H. Khanpour, B. A. Kniehl, and M. Soleymaninia, $B$-hadron fragmentation functions at next-to-next-to-leading order from a global analysis of $e^{+} e^{-}$annihilation data, Phys. Rev. D 99, 114001 (2019).

[24] J. Abdallah et al. (DELPHI Collaboration), A study of the $b$-quark fragmentation function with the DELPHI detector at LEP I and an averaged distribution obtained at the $Z$ Pole, Eur. Phys. J. C 71, 1557 (2011).

[25] M. Fischer, S. Groote, J. G. Korner, M. C. Mauser, and B. Lampe, Polarized top decay into polarized $W: t(\uparrow) \rightarrow$ $W(\uparrow)+b$ at $O\left(\alpha_{s}\right)$, Phys. Lett. B 451, 406 (1999).

[26] M. Fischer, S. Groote, J. G. Körner, and M. C. Mauser, Complete angular analysis of polarized top decay at $O\left(\alpha_{S}\right)$, Phys. Rev. D 65, 054036 (2002).

[27] W. Bernreuther, P. González, and C. Mellein, Decays of polarized top quarks to lepton, neutrino, and jets at NLO QCD, Eur. Phys. J. C 74, 2815 (2014).

[28] A. Czarnecki, S. Groote, J. G. Körner, and J. H. Piclum, NNLO QCD corrections to the polarized top quark decay $t(\uparrow) \rightarrow X_{b}+W^{+}$, Phys. Rev. D 97, 094008 (2018).

[29] N. Cabibbo, Unitary Symmetry and Leptonic Decays, Phys. Rev. Lett. 10, 531 (1963).

[30] M. Kobayashi and T. Maskawa, $C P$-violation in the renormalizable theory of weak interaction, Prog. Theor. Phys. 49, 652 (1973).

[31] B. A. Kniehl, A. V. Kotikov, A. I. Onishchenko, and O. L. Veretin, Strong-Coupling Constant with Flavor Thresholds at Five Loops in the Modified Minimal-Subtraction Scheme, Phys. Rev. Lett. 97, 042001 (2006). 